\title{
Synthesis of Mesoporous Silica Nanowires and Their Application in Enzyme Immobilization
}

\author{
Peng Liu ${ }^{*}$, Xinlong $\mathrm{Liu}^{1}, \mathrm{Na} \mathrm{An}^{1}$, and Peng Wang ${ }^{1}$ \\ ${ }^{1}$ School of Biological Engineering, Tianjin Modern Vocational Technology College, Tianjin, 300350, China
}

\begin{abstract}
Hydrophobic mesoporous silica nanowires were synthesis and then employed as support for immobilization of lipase from Candida antarctica via covalent bonding (CALB@MSW). The parameters were optimized and the optimum conditions were as follows: GA concentration $5.5 \mathrm{wt} \%$, activation time $60 \mathrm{~min}$ and CALB concentration $4 \mathrm{mg} / \mathrm{mL}$. Under these conditions, the protein loading and specific activity of CALB@MSW were $138.3 \mathrm{mg} / \mathrm{g}_{\text {support }}$ and $41.1 \mathrm{U} / \mathrm{mg}_{\text {support}}$, respectively. Compared with free CALB, CALB@MSW showed better thermal stability and pH stability. The maximum yield of biodiesel catalytic by CALB@MSW was 93.4\%. After reused 8 times, CALB@MSW still remained $95.75 \%$ initial activity showing better stability than free CALB.
\end{abstract}

\section{Introduction}

Lipase possesses very broad substrate specificity and has extensively utilized in chemical and pharmaceutical industry to produce high value-added chemicals, biosensors and drugs. However, lipase only exhibits efficient activity and selectivity in mild conditions and will be devitalized once encountered extreme conditions ( $\mathrm{pH}$ and temperature), due to the changes of conformation or other transformations of stereo chemical structure. Meanwhile, lipase often suffers limitation in broader application because of its poor reusability leading to high cost. One of approaches to overcome these disadvantages is to immobilize lipase onto/into a support, improving reactivity and stability ${ }^{[1]}$.

Many materials have explored as a support for immobilization, such as zeolite, resin, sol-gels, polymeric hydrogels, mesoporous carbon, organic microparticles and controlled pore glass ${ }^{[2]}$. Whereas, recently, mesoporous templated silicates and its related composites have received much attention with their abundant porosity, wide size distribution, controllable shape and morphology, diversified composition and high chemical/thermal/ process reusability ${ }^{[3]}$. Mesoporous silica nanowires (MSW) with three-dimensional (3D) network and hierarchical pore structures are an outstanding representative. Their applications in catalysis have aroused extensive research interest due to their considerable advantages, including high surface area, mechanical stability and favorable mass transport taking place in both reaction and sorption processes benefitting from the multimodal pore systems ${ }^{[4]}$. Furthermore, integration of various functional groups in MSW by changing the precursor can create a comfortable catalytic microenvironment for lipase and thus further improved the performance of the biocatalyst $\mathrm{t}^{[5]}$.
In this work, we develop a synthetic route to prepare the functional mesoporous silica nanowires. Then the MSW are used as a catalyst support for the immobilization of Candida antarctica lipase B (CALB) and tested for biodiesel production to explore their applicability. The optimum synthesis conditions of the catalyst were studied based on the yield of biodiesel.

\section{Materials and Methods}

\subsection{Material}

Glutaraldehyde (GA), oleic acid, methanol and hexadecyltrimethylammonium chloride (CTACl) were purchased from Tianjin Chemical Corporation (Tianjin, China). 3-amino-propyltriethoxysilane 98\% (APTES) and1,2-bis(trimethoxysilyl)ethane (BTME) were purchased from Sigma Chemical Co (St. Louis, MO, USA). Candida antarctica lipase B and N435 were purchased from Beijing Cliscent Technology Co., Ltd. All other chemicals and reagents were of analytical grade.

\subsection{Preparation of MSW}

Functional MSW were synthesized by hydrolysis of BTME with sodium hydroxide as an alkaline source. Firstly, distilled water $(13 \mathrm{~mL})$, methanol (1 mL), CTACl aqueous solution $(25 \%, 2 \mathrm{~mL})$ and sodium hydroxide $(0.5$ $\mathrm{mL})$ were mixed in a pressure flask and then stirred at room temperature for $30 \mathrm{~min}$. Secondly, $1.5 \mathrm{~mL}$ of BTME was slowly added. After that, the mixture was heated in drying oven at $70{ }^{\circ} \mathrm{C}$ for $1.5 \mathrm{~h}$. The opaque precipitates (MSW) were obtained by adding ethanol and centrifuged at $10000 \mathrm{rpm}$ for $1.5 \mathrm{~min}$.

* Corresponding author: wangp20@yeah.net (P. W.) 


\subsection{Immobilization of CALB}

CALB was immobilized to MSW by covalent bond with GA as crosslinker. $100 \mathrm{mg}$ of MSW was suspended in $5 \mathrm{~mL}$ of phosphate buffer solution (PBS, $10 \mathrm{mM}, \mathrm{pH} 7.0$ ) containing $0.2 \%$ GA. The mixture was magnetically stirred for $1 \mathrm{~h}$ at $30^{\circ} \mathrm{C}$ and then centrifuged. The sediment after washed with distilled water three times was dispersed into CALB solution $(0.2 \mathrm{mg} / \mathrm{mL}$ PBS $)$ under stirring for $30 \mathrm{~min}$. Then, the mixture was centrifuged and washed three times with buffer solution. The obtained solid (named CALB@MSW) was freeze-dried and stored at 4 ${ }^{\circ} \mathrm{C}$ until it was used.

The effect of the preparation parameters (GA concentration, active time and CALB concentration) on the performance of CALB@MSW was investigated in the general procedure.

\subsection{Evaluation of the activity and stability}

The activity of CALB@MSW was evaluated in the enzymatic reactions with oleic acid and methanol as substrates to produce biodiesel. For esterification reactions, $0.1 \mathrm{~mol}$ of methanol, $0.1 \mathrm{~mol}$ of oleic acid and $50 \mathrm{mg}$ of catalyst were added to the flask containing $2 \mathrm{~mL}$ cyclohexane. The mixture was shaken $(200 \mathrm{r} / \mathrm{min})$ at room temperature for $8 \mathrm{~h}$ and then centrifuged at $10000 \mathrm{r} / \mathrm{min}$ for $1 \mathrm{~min}$. The biodiesel yield was calculated after the analysis of supernate using gas chromatography ${ }^{[6]}$.

The thermal stability of CALB@MSW and free CALB were analysed by measuring the activity change after being incubated in cyclohexane at $60^{\circ} \mathrm{C}$ or $70^{\circ} \mathrm{C}$ for $60 \mathrm{~h}$. The pH stability of CALB@MSW and free CALB were evaluated by measuring the activity change after being incubated in a solution (pH 4.0 and 7.0) for $72 \mathrm{~h}$.

\section{Results}

\subsection{The Effect of GA Concentration upon CALB@MSW}

The GA concentration has a significant effect on the properties of CALB@MSW, as shown in Fig. 1. When the GA concentration increased from $4.0 \%$ to $6.5 \%$, the protein loading of MSW increased from $90 \mathrm{mg} / \mathrm{g}_{\text {support }}$ to $129 \mathrm{mg} / \mathrm{g}_{\text {support }}$, indicating that the more GA modification on MSW, the more conducive to CALB molecules fixation. However, the increasing trend of protein loading decreased gradually, mainly due to the limited number of CALB in the solution, which restricted the immobilization process. Fig. 1 also showed the effect of GA concentration on the specific activity of CALB@MSW. With the increase of the concentration of GA to $5.5 \mathrm{wt} . \%$, the specific activity of CALB@MSW also increased rapidly, and soon reached the peak activity of $34.2 \mathrm{U} / \mathrm{mg}_{\text {support }}$. As the GA concentration further increased, the specific activity of CALB@MSW began to decrease. This might be because a large number of CALB clustered on the surface or in the pores of MSW and interfere with each other, which affected the catalytic activity and mass transfer. Therefore, $5.5 \%$ GA concentration was the best choice for the preparation of CALB@MSW.

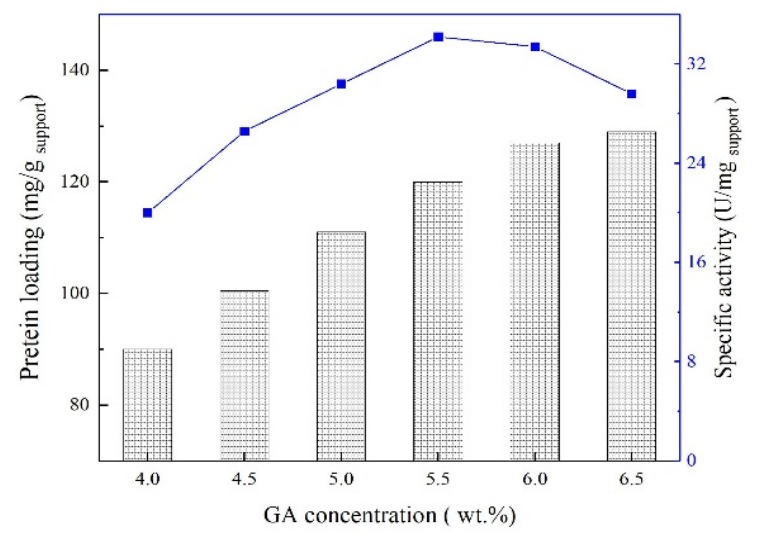

Fig. 1 The effect of GA concentration on the specific activity and protein loading of CALB@MSW (specific activity: point plot, protein loading: histogram)

\subsection{The Effect of activation time upon CALB@MSW}

Fig. 2 revealed the effects of activation time on the activity of CALB@MSW. With the extension of activation time from $30 \mathrm{~min}$ to $90 \mathrm{~min}$, the specific activity of CALB@ MSW increased first and then decreased. This was because the short activation time resulted in insufficient amount of aldehyde groups on MSW and limited the loading amount of CALB leading to lower activity ${ }^{[7]}$. Although longer activation time could provide more binding sites increasing protein loading and apparent activity, it could also destroy the CALB structure or promote the occurrence of polymerization leading to the reducing of specific activity of CALB@MSW ${ }^{[8]}$. In conclusion, the optimal activation time was $60 \mathrm{~min}$.

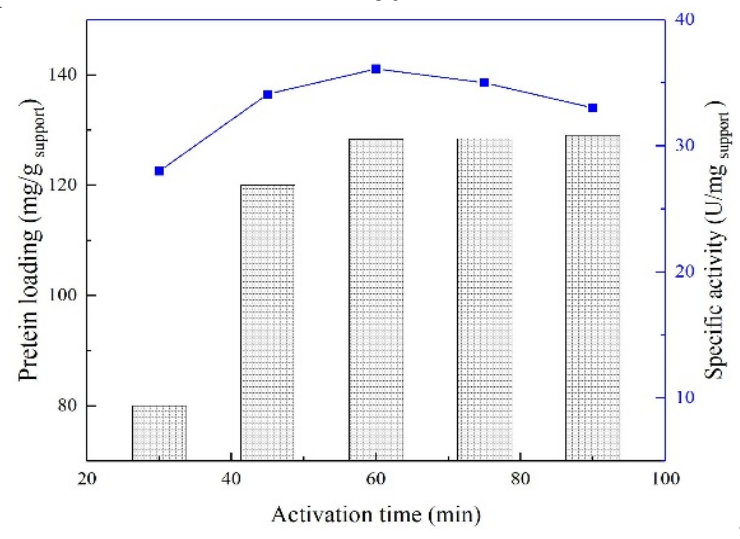

Fig.2 The effect of activation time on the protein loading and specific activity of CALB@MSW (specific activity: point plot, protein loading: histogram)

\subsection{The Effect of CALB concentration upon CALB@MSW}

In order to further optimize the preparation conditions of CALB@MSW, the effect of initial CALB concentration on the protein loading and specific activity was investigated. According to Fig.3, protein loading and specific activity of CALB@MSW both increased with the increasing of CALB concentration. When CALB 
concentration increased to $4.0 \mathrm{mg} / \mathrm{mL}$, the protein loading of and specific activity reached the maximum and remained unchanged. This was attributed to the limited number of GA residue on MSW leading to a maximum protein loading and specific activity. In addition, the slight decrease of CALB@MSW activity might be attributed to the mutual occlusion of active sites and increased steric hindrance resulting from excessive CALB loading. Therefore, the optimal CALB concentration preparing CALB@MSW was $4.0 \mathrm{mg} / \mathrm{mL}$, and its protein loading and specific activity were $138.3 \mathrm{mg} / \mathrm{g}_{\text {support }}$ and 41.1 $\mathrm{U} / \mathrm{mg}_{\text {support }}$, respectively.

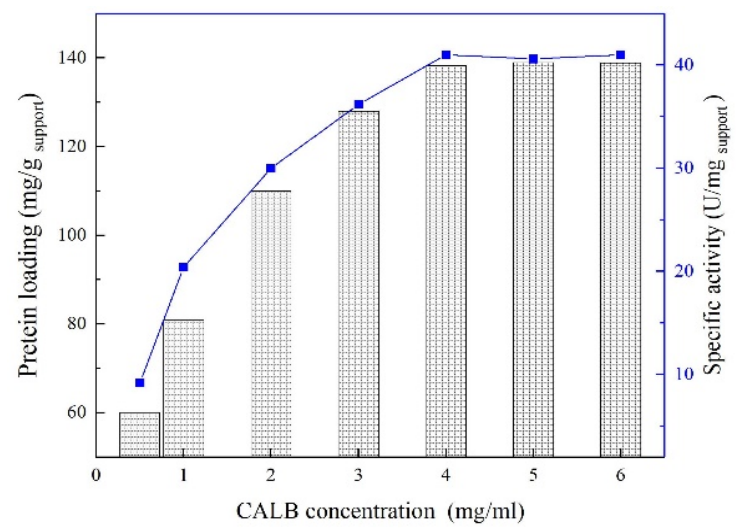

Fig.3 The effect of CALB concentration on the protein loading and specific activity of CALB@MSW (specific activity: point plot, protein loading: histogram)

\subsection{The thermal stability of CALB@MSW}

Fig.4 showed the stability of CALB@MSW and free CALB in cyclohexane at $60{ }^{\circ} \mathrm{C}$ and $70{ }^{\circ} \mathrm{C}$. The initial activity was defined as $100 \%$. After $60 \mathrm{~h}$ of incubation at $60{ }^{\circ} \mathrm{C}$, the relative activity of CALB@MSW was sharply reduced to $51 \%$, and the free CALB was reduced to $23 \%$ of its initial activity. After incubation at $70{ }^{\circ} \mathrm{C}$ for $60 \mathrm{~h}$, the relative activities of CALB@MSW and free CALB were $40 \%$ and $11 \%$, respectively. The higher residual activity and slower downward trend of activity proved that CALB@MSW owned better thermal stability than free CALB. The main reason for the decrease of activity was that the CALB molecules were prone to thermal denaturation at high temperature, which changed their conformation $^{[9]}$. But for CALB@MSW, the covalent bonding between CALB and MSW and the relatively "crowded" microenvironment for the resulting from the folded pores in MSW both could limit the conformation change of the immobilized CALB molecules, thus maintaining good thermal stability compared to free $\mathrm{CALB}^{[3]}$.

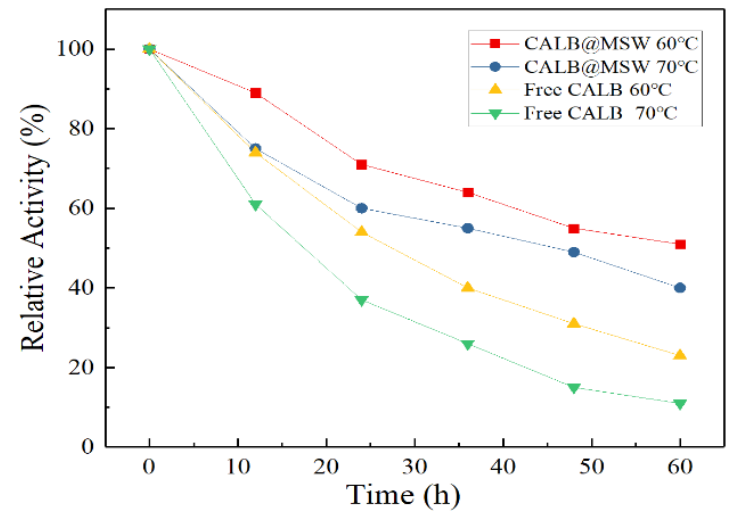

Fig.4 The thermal stability of CALB@MSW and free CALB at $60{ }^{\circ} \mathrm{C}$ and $70{ }^{\circ} \mathrm{C}$

\subsection{The pH stability of CALB@MSW}

The solution $\mathrm{pH}$ also had an important effect on the activity and stability of CALB@MSW, and the degree mainly depended on the type of enzyme, the immobilized method and the type of carrier. Fig.5 analysed the effects of different $\mathrm{pH}(3.0-12.0)$ on the activities of CALB@MSW and free CALB.

The results showed that the highest activity of free CALB was obtained at $\mathrm{pH} 8.0$, defining as $100 \%$. Any deviation from this $\mathrm{pH}$ would lead to a rapid decline in activity. For example, only $21.2 \%$ of the maximum activity was maintained at $\mathrm{pH} 3$, while only $14.1 \%$ of the relative activity was retained at $\mathrm{pH} 12$. This was because that free CALB was exposed to the solution and was directly affected by the $\mathrm{pH}$ of the system. Based on this, the dissociation state of groups in the CALB and substrate molecule were easily changed, and thus affecting the molecular conformation, binding state with substrates and catalytic performance of CALB. On the contrary, the activity of CALB@MSW showed the maximum catalytic activity in the range of $\mathrm{pH} 6.0$ to 9.0 . When the $\mathrm{pH}$ deviated from this optimal range, the activity of CALB@MSW would also be adversely affected and decreased, but the trend of change was much slower than that of free CALB. When the $\mathrm{pH}$ was 3 and 12, the relative activity was still $79 \%$ and $82.3 \%$, respectively, indicating that CALB@MSW had better pH stability. This result was consistent with the literature reports that the optimal $\mathrm{pH}$ range of immobilized enzymes was generally wider than that of free enzymes ${ }^{[10]}$.

\subsection{The reusability of CALB@MSW}

Biodiesel, series of fatty acid alkyl esters, was an ideal alternative fuel for diesel due to its biodegradable, nontoxic, low emission profile and renewable features. However, the industrialization of enzymatic biodiesel production was often hampered by the high cost and poor operational stability of enzymes. These drawbacks could 


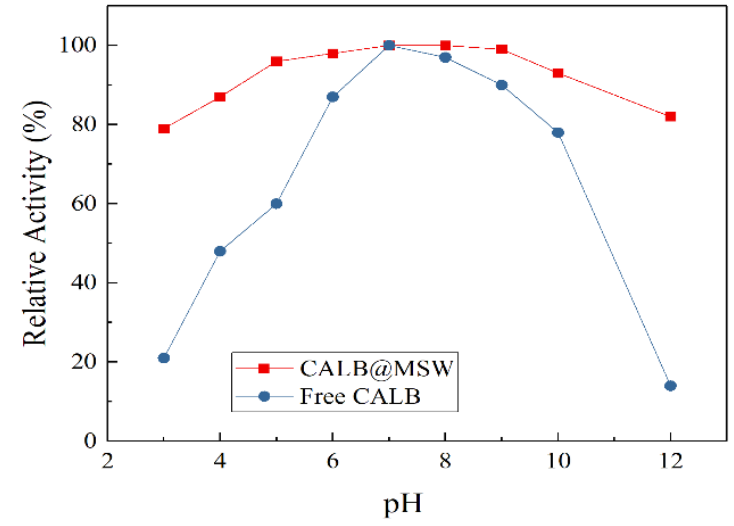

Fig.5 The pH stability of CALB@MSW and free CALB

generally be overcome by the immobilization of lipase. Therefore, the reusability of CALB@MSW was investigated in the synthesis of biodiesel and compared to free CALB.

Fig. 6 showed the catalytic performance of CALB@MSW and free CALB in biodiesel production, the maximum yield of biodiesel (based on oleic acid) were $93.4 \%$ and $81.4 \%$ for the first using time of CALB@MSW and free CALB after $24 \mathrm{~h}$. When CALB@MSW was reused for 8 times, the biodiesel yield was $89.45 \%$, which was equivalent to remain $95.75 \%$ initial activity. Nevertheless, the yield catalyzed by free CALB under the same operating conditions decreased dramatically to $5.34 \%$, which might be due to the destruction of the CALB structure by shear force and adverse factors in the reaction system. CALB@MSW owned better catalytic performance and reusability than free CALB, mainly arised by the protection offered by MSW and the stable covalent linkage. Additionally, the hydrophobic group on MSW surface provided a relatively hydrophobic microenvironment for CALB, which improved the catalytic activity and stability and inhibited the damage of methanol to CALB to a certain extent ${ }^{[11]}$. As for the biodiesel yield reduction, it might be due to the loss of CALB@MSW during recovery.

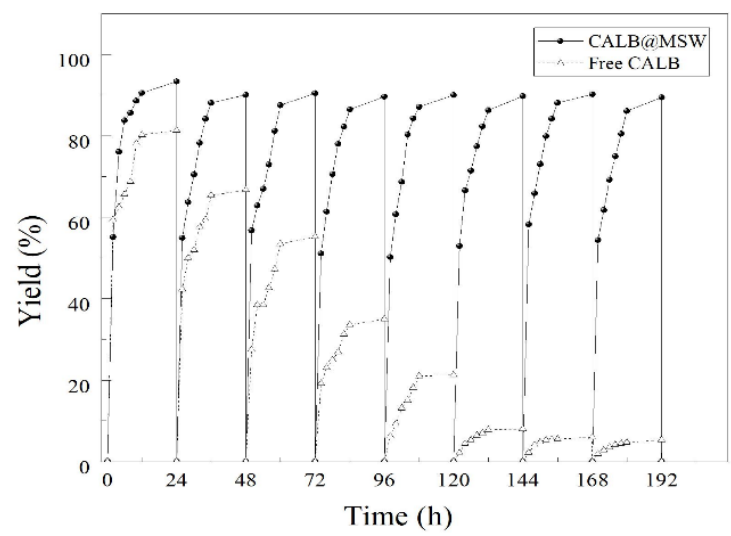

Fig.6 The catalytic performance of CALB@MSW and free CALB in biodiesel production

\section{Conclusion}

The immobilization of CALB in hydrophobic mesoporous silica nanowires via covalent bonding was systematically studied. The preparation conditions of CALB@MSW, including GA concentration, activation time and CALB concentration, were optimized by single factor analysis with the specific activity and protein loading of CALB@MSW as evaluation indices. Compared with free CALB, CALB@MSW showed better thermal stability and $\mathrm{pH}$ stability. In enzymatic biodiesel production, CALB@MSW showed better activity and reusability than free CALB demonstrating the value of immobilization.

\section{References}

1. L. Wang, X. Liu, Y. Jiang, L. Zhou, L. Ma, Y. He and J. Gao. Catalysts 8, 587 (2018)

2. P. Periyat and E. Magner. 65-74 (2011)

3. S. Hudson, J. Cooney and E. Magner. Angewandte Chemie International Edition 47, 8582-8594 (2008)

4. N. Li, J.-G. Wang, H.-J. Zhou, P.-C. Sun and T.-H. Chen. Chemistry of Materials 23, 4241-4249 (2011)

5. M. Petkar, A. Lali, P. Caimi and M. Daminati. Journal of Molecular Catalysis B: Enzymatic 39, 83-90 (2006)

6. H. Gu, Y. Jiang, L. Zhou and J. Gao. Energy Environ. Sci. 4, 1337-1344 (2011)

7. J. Gao, H. Yu, L. Zhou, Y. He, L. Ma and Y. Jiang. Biochemical Engineering Journal 117, 92-101 (2017)

8. O. Barbosa, C. Ortiz, R.T. Ángel Berenguer-Murcia, Rafael C. Rodrigues and R. Fernandez-Lafuente. RSC Advances 4, 1583-1600 (2014)

9. B. Singh, G. Bulusu and A. Mitra. The Journal of Physical Chemistry B 119, 392-409 (2015)

10. A. Karout, C. Chopard and A.C. Pierre. Journal of Molecular Catalysis B: Enzymatic 44, 117-127 (2007)

11. Q. Jin, G. Jia, Y. Zhang, Q. Yang and C. Li. Langmuir 27, 12016-12024 (2011) 